ACT-07/95

CERN-TH/95-88

CTP-TAMU-21/95

UMN-TH-1349/95

SNUTP 95-041

hep-ph/9505438

\title{
On the Thermal Regeneration Rate for Light Gravitinos in the Early Universe
}

\author{
John Ellis ${ }^{1}$, D.V. Nanopoulos ${ }^{2,3}$, Keith A. Olive ${ }^{4}$ and Soo-Jong Rey ${ }^{5}$ \\ Theory Division, CERN, CH-1211 Geneva SWITZERLAND ${ }^{1}$ \\ Department of Physics, Texas A\&M University, College Station, TX 77843 USA ${ }^{2}$ \\ Astroparticle Physics Group, Houston Advanced Research Center (HARC), \\ The Mitchell Campus, The Woodlands, TX 77381, USA ${ }^{3}$ \\ Department of Physics, University of Minnesota, Minneapolis MN 55455 USA $^{4}$ \\ Physics Department and Center for Theoretical Physics, Seoul National University, Seoul \\ 151-742 KOREA ${ }^{5}$
}

\begin{abstract}
We investigate the light gravitino regeneration rate in the early Universe in models based on $N=1$ supergravity. Motivated by a recent claim by Fischler, we evaluate finite-temperature effects on the gravitino regeneration rate due to the hot primordial plasma for a wide range of the supersymmetrybreaking scale $F$. We find that thermal corrections to the gravitino pole mass and to the Goldstino coupling are negligible for a wide range of temperatures, thereby justifying the extension of the equivalence theorem for the helicity-1/2 gravitino and Goldstino to a hot primordial plasma background.
\end{abstract}


Utilizing the Braaten-Pisarski resummation method, the helicity-1/2 gravitino regeneration rate is found to be $0.25 \alpha_{s}(T) \log \left(1 / \alpha_{s}(T)\right)\left|m_{\text {soft }} / F\right|^{2} T^{3}(1+$ $\left.\alpha_{s}(T) \log \left(1 / \alpha_{s}(T)\right)+T^{2} /|F|\right)$ up to a calculable, model-dependent $\mathcal{O}(1)$ numerical factor. We review the implications of this regeneration rate for supergravity cosmology, focusing in particular on scenaria for baryogenesis.

\section{INTRODUCTION}

One of the most serious cosmological constraints in the framwework of $N=1$ supergravity is the gravitino problem [1] [2]. Whilst inflation dilutes exponentially any pre-existing gravitinos, thermal gravitinos may be regenerated in significant numbers if the post-inflationary reheating temperature is sufficiently high. Once regenerated, thermal gravitinos may lead to a cosmological disaster. Long-lived gravitinos may dominate the energy density of the early Universe, or their decays may alter the light-element abundances calculated in primordial nucleosynthesis, if the gravitinos decay after nucleosynthesis. Therefore, any successful supersymmetric inflationary model should keep the reheating temperature low enough that sufficiently few gravitinos are regenerated subsequently. This requirement is known to put a severe upper bound on the reheating temperature [3]- [12], unless the gravitino is very light.

At energies that are sufficiently high compared to the gravitino mass, it is known that interactions of the helicity- $1 / 2$ components of the gravitino dominate over the helicity-3/2 components. Fischler [10] recently re-analyzed the gravitino regeneration rate and suggested that heat-bath effects might enhance greatly this rate at high temperature. Fischler's argument was based on the well-known fact that supersymmetry (SUSY) is broken [13] at finite temperature [14] [15], because the thermal distributions are different for bosons 
and fermions. He argued that the gravitino regeneration rate should be proportional to $T^{5}$, instead of $T^{3}$ as estimated previously [4]. If correct, his result would imply that the reheating temperature should not exceed $\sim 10^{4}-10^{7}$ $\mathrm{GeV}$, or the gravitino should be lighter than $1 \mathrm{KeV}$ or heavier than several TeV. Such a conclusion would be significant for both cosmology and supersymmetric phenomenology, requiring either a low reheating temperature or an extremely low/high gravitino mass, with a correspondingly low/high supersymmetry breaking scale $\sqrt{|F|} \sim 10^{6} / 10^{11} \mathrm{GeV}$. Motivated by these considerations, in this paper we study finite-temperature effects on the gravitino regeneration rate in a more systematic way.

At finite temperature there are various thermal effects one has to take into account in order to understand the chemical equilibration of light gravitinos. First, the heat bath may generate temperature-dependent mass renormalization. Since the gravitinos are light, one might worry that the thermal mass renormalization effect could be significant. If the thermal mass renormalization is so large that it exceeds the typical energy of the gravitino or the Goldstino-matter coupling strength becomes strong, then one cannot expect any more that the helicity-1/2 components of gravitinos are dominant in regeneration processes. Secondly, the reaction rate for chemical equilibration has to be calculated using the full panoply of thermal field theory. The Landau damping phenomena in the electron-gas plasma [16] and hot gauge theories are well-known examples. Finite-temperature effects enter through the effective thermal propagators, vertices and through the external particles' thermal phase space factors. In this paper, we adopt the method proposed by Braaten and Pisarski [17] for taking these effects correctly into account, and calculating consistently thermal reaction rates. In this method, the underlying spontaneously-broken supersymmetry may not be manifest. Not only does the heat bath distinguish bosons and fermions through their different equilibrium 
distribution functions, but also intrinsically finite-temperature effects such as collective excitations are more significant for fermions and gauge bosons than for scalar particles. However, our explicit calculations show that thermal effects are quite insignificant, in that the finite-temperature corrections to the gravitino mass and to the Goldstino-matter coupling are negligible, and the zero-temperature estimates of the gravitino regeneration rate are qualitatively correct. This conclusion is in disagreement with Fischler's argument.

This paper is organized as follows. In Section 2, we review the low-energy effective Lagrangian for the helicity-3/2 and $-1 / 2$ components of a light gravitino. Then we calculate temperature-dependent corrections to the gravitino mass, and show that they are proportional to $\left(T / M_{P}\right)^{2} m_{\text {soft }}$, where $m_{\text {soft }}$ represents a typical soft supersymmetry-breaking mass splitting for a chiral or gange supermultiplet. Similarly, we find a thermal correction to the Goldstino-matter coupling due to thermal Goldstino dynamics that is proportional to $T^{2} /|F|$. These corrections are numerically negligible for the range of supersymmetry-breaking scale $\sqrt{|F|}$ of phenomenological interest. Therefore we may continue to use the equivalence theorem for light helicity-1/2 gravitinos and Goldstinos. In Section 3, utilizing kinetic theory and diagrammatics [18], we present a reaction-rate formula valid when the plasma is slightly out of chemical equilibrium. In Section 4, we calculate the gravitino regeneration rate, applying the effective Lagrangian for light gravitinos obtained in Section 2 and the finite-temperature formalism given in Section 3. In Section 4 the regeneration rate is expressed as a discontinuity of the Goldstino self-energy across an appropriate kinematical cut relevant to the Goldstino regeneration process. For a wide temperature range, including that of phenomenological interest to us, we find that the regeneration rate is qualitatively the same as the estimate made at zero-temperature [6,12]. Compared to the zero-temperature rate, the finite-temperature corrections contribute $\sim \log \left(1 / \alpha_{s}(T)\right)$, which re- 
sults from finite-temperature effects at temperature between $\sim g T$ and $\sim T$. Next-order corrections are proportional to $\alpha_{s}(T) \log \left(1 / \alpha_{s}(T)\right)$, mainly from thermal QCD corrections to the Goldstino-matter coupling vertex. Therefore we conclude that thermal effects do not alter drastically the temperature dependence of the reaction rate as Fischler claimed. In Section 5, we discuss the implications of our results for cosmology focusing in particular on refined treatment of reheating dynamics and implications to the baryogenesis, and in Section 6 we summarize our results.

\section{LOW-ENERGY EFFECTIVE LAGRANGIAN FOR LIGHT GRAVITINOS}

We first recapitulate the relevant effective Lagrangian for light gravitinos [19], in which supersymmetry is broken by the standard super-Higgs effect in $N=1$ supergravity theory. Since the gravitino interacts with gravitational strength, the relevant terms in the $N=1$ supergravity Lagrangian are always suppressed at least by one power of Planck scale $M_{P} \equiv 1 / \sqrt{G}_{N}$. We denote the chiral and gauge supermultiplets by $(\phi, \chi)$ and $\left(A_{\mu}, \lambda\right)$. Keeping only the leading dimension-5 terms, the relevant Lagrangian for an on-shell gravitino in the $\gamma^{\mu} \psi_{\mu}=0$ gange is given by

$\frac{L}{\sqrt{-g}}=\frac{1}{\sqrt{2} M_{P}}\left[D_{\mu} \overline{\phi \psi}_{\nu} \gamma^{\mu} \gamma^{\nu} \chi_{R}+D_{\mu} \bar{\phi}_{\chi_{L}} \gamma^{\nu} \gamma^{\mu} \psi_{\nu}+\frac{1}{\sqrt{2}} \bar{\psi}_{\mu} \sigma^{\nu \lambda} \gamma^{\mu} \lambda F_{\nu \lambda}\right]+$ h.c.

When combined with a globally-supersymmetric Lagrangian for matter and gauge multiplets, the above Lagrangian may be used to calculate processes involving the helicity-3/2 components of light gravitinos.

In addition to the helicity-3/2 components, the super-Higgs effect from spontaneously-broken supersymmetry gives rise to helicity-1/2 Goldstino components. As long as the typical energy-momentum scale of the gravitino is much larger than its mass $m_{3 / 2}$ and $m_{3 / 2}$ is not larger than the effective 
mass splitting of Bose and Fermi components for gauge and matter multiplets ( $m_{3 / 2}$ and the mass splittings may be decoupled as in the case of no-scale supergravity [20]), one can treat the helicity-1/2 components of gravitinos as true Goldstinos. This is the situation we study in what follows, where we consider matter and gauge supermultiplet mass splitting of order the weak scale, with a gravitino that could be lighter. More explicitly, in models in which supersymmetry is broken by a hidden-sector field $S$ at a scale $\langle S\rangle_{\theta^{2}} \equiv F \neq 0$, and is coupled to the observable sector at an intermediate-scale $M$, the soft supersymmetry breaking mass splittings are generated by the D term

$$
\frac{1}{M^{2}} \int d^{2} \theta d^{2} \bar{\theta}^{2} S \bar{S} \Phi \bar{\Phi}
$$

and by the F term

$$
\frac{1}{M} \int d^{2} \theta S W_{\alpha} W^{\alpha}+\text { h.c. }
$$

Therefore, mass splittings are of order

$$
\Delta m^{2} \approx\left(\frac{|F|}{M}\right)^{2} ; \quad m_{\lambda} \approx \frac{F}{M} .
$$

For supersymmetry to be relevant to the stability of the weak scale, the soft supersymmetry-breaking mass splittings should be of the same order as the weak scale $M_{W}$. For simplicity, we denote both of these soft SUSY breaking masses by a common mass $m_{\text {soft }}$. In this case, the gravitino mass is given by

$$
m_{3 / 2}=\frac{|F|}{\sqrt{6} M_{P}} \approx \frac{M}{M_{P}} m_{\text {soft }} .
$$

We consider $m_{3 / 2}$ no larger than $m_{\text {soft }}$, corresponding to $M$ no larger than $M_{P}$

In the situation of interest to us, for which $m_{3 / 2}$ is much smaller than the typical energy-momentum scale of the gravitino, the low-energy theorem for the Goldstino implies that the helicity-1/2 components of gravitinos are described by 


$$
\psi_{\mu}=\sqrt{\frac{2}{3}} \frac{1}{m_{3 / 2}} i \partial_{\mu} \psi
$$

where $\psi$ denotes the Goldstino and $\sqrt{2 / 3}$ comes from the spin-1 ClebschGordan coefficient.

Substitution of Eq.(6) into Eq.(1) converts the dimension-5 operators of gravitinos into dimension- 6 operators involving Goldstinos. These dimension6 operators in turn yield bad high-energy behavior of the Goldstinos compared to the underlying supergravity theory. As such the leading divergences of the helicity-1/2 gravitino-gravitino scattering amplitude present in each individual diagram must cancel out in the total amplitude. This is a consequence of the fact that the helicity-1/2 components of gravitinos are unphysical in the limit where supersymmetry is unbroken and realized linearly. It is desirable to rearrange the effective Lagrangian so that this fact is manifest. In terms of Feynman diagrams this is done by integrating by parts and using equations of motion for external gauge and matter multiplet lines and subtracting the leading divergence from each individual diagram for internal gauge and multiplet lines. After this is done, the interactions of the Goldstino with chiral and gauge supermultiplets are given by

$$
L=-\frac{m_{s o f t}}{8 F} \bar{\psi}\left[\gamma_{\mu}, \gamma_{\nu}\right] \lambda F^{\mu \nu}+\frac{\sqrt{2} m_{s o f t}^{2}}{F}\left(\bar{\chi}_{R} \psi\right) \phi+h . c .
$$

The Lagrangian depends explicitly on the soft supersymmetry breaking masses $m_{\text {soft }}$, hence, on the Goldstino-matter coupling constant $g_{G}=$ $m_{\text {soft }}^{2} /|F|$. We emphasize again that the Goldstino-matter coupling $g_{G}$ is nonzero only for softly-broken supersymmetry.

At finite temperature, two questions should be addressed regarding the effective Lagrangian Eq.(7). First, the gravitino mass may receive thermal corrections. If this correction is significant, one cannot apply the low-energy theorem to evaluate the leading-order interactions of gravitinos. We have 
calculated the finite-temperature correction to the gravitino mass using the interaction vertices in Eq.(1). In the imaginary-time formalism of thermal field theory, the correction is given in a form $\bar{\psi}_{\mu} \Sigma^{\mu \nu}(T) \psi_{\nu}$, where $\Sigma^{\mu \nu}(T)$ denotes the two self-energy diagrams at finite temperature depicted in Figure 1. An explicit calculation based on the dimension-5 operators in Eq.(1) shows that the thermally-induced gravitino mass is given by

$$
\frac{1}{2} \operatorname{Tr}\left(\sigma_{\mu \nu} \Sigma^{\mu \nu}(T)\right) \approx \frac{\pi^{2}}{12} \frac{T^{2}}{M_{P}^{2}} m_{\text {soft }}
$$

The manifest chiral invariance present in unbroken supergravity forbids a thermally-induced gravitino mass for $m_{\text {soft }}=0$. This underlies the dependence on the soft SUSY breaking mass $m_{\text {soft }}$ in Eq.(8). Comparing the result to $m_{3 / 2}$, we see that the thermal correction is completely negligible as long as $T \ll \sqrt{M M_{P}}$. Even in models with $M \sim 10 \mathrm{TeV}$, the minimum temperature needed to obtain an appreciable thermal mass correction is $\sim 10^{11} \mathrm{GeV}$.

Secondly, the Goldstino-matter coupling may receive thermal corrections as depicted in Figure 2. Recall that $g_{G}=m_{\text {soft }}^{2} /|F|$ depends explicitly on the soft supersymmetry breaking masses $m_{\text {soft }}$. Therefore, thermal corrections to $m_{\text {soft }}$ and $F$ may in turn induce thermal corrections to the coupling $g_{G}$. One might expect that the soft supersymmetry breaking mass $m_{s o f t}^{2}$ receives a thermal correction of the form $m_{\text {soft }}^{2} \rightarrow m_{\text {soft }}^{2}+\mathcal{O}\left(T^{2}\right)$. This in turn might induce $\sim T^{2}$ thermal correction to the coupling $g_{G}$ as in the Fischler's evaluation of the reaction rate. However, these steps are questionable. First, even for the plasma heat bath, the interaction of the gravitino with other matter is described by a Noether coupling of the form $\psi_{\mu} S^{\mu}+h . c$. Secondly, the soft masses $m_{\text {soft }}$ are generated by the interactions Eqs. $(2,3)$ in the form of Eq.(4). This tells us that, for a fixed intermediate scale $M$, thermal corrections to $g_{G}$ from the Goldstino dynamics itself mainly originate from thermal corrections to the supersymmetry breaking F-term $F=\langle S\rangle_{\theta^{2}}$. 
There are actually two sources of thermal corrections to $g_{G}=m_{\text {soft }}^{2} /|F|$. The first is the thermal mass correction to $m_{\text {soft }}$ due to the Goldstino dynamics. At the one loop level, the Goldstino interaction Eq.(7) yields

$$
m_{\text {soft }}^{2}(T) \approx m_{\text {soft }}^{2}\left(1-\frac{1}{24} \frac{T^{2}}{|F|}\right)
$$

At the same order, the $\mathrm{F}$ term receives a thermal correction from the thermal Goldstino wave-function renormalization

$$
|F(T)| \approx|F|\left(1-\frac{1}{48} \frac{T^{2}}{|F|}\right)
$$

Thermal tadpole corrections from interactions in Eqs.(2,3) only give rise to $|\Delta F(T)| \approx T^{2} / M^{2}$, and hence are negligible as long as $|F| \approx M \cdot M_{W} \ll M^{2}$. Therefore, we find thermal Goldstino dynamics induces a correction of size

$$
g_{G}(T) \approx g_{G}\left(1-\frac{1}{48} \frac{T^{2}}{|F|}+\cdots\right)
$$

Again, the correction Eq.(11) is numerically negligible for temperature of interest to us: $T \ll \sqrt{|F|}$. The actual thermal Goldstino correction might even be further suppressed if there is a direct extension of the finite-temperature nonrenormalization of the Goldberger-Treiman relation [21] for the chiral dynamics of pions and nucleons to the Goldstino dynamics of spontaneouslybroken supersymmetry.

Additional corrections to the $g_{G}$ coupling come from the gauge interactions. Unlike the thermal correction from the Goldstino dynamics, such corrections from gauge interactions may be taken into account self-consistently using the effective Lagrangian Eq.(7) and the resummed perturbation method. Through explicit calculation in Section 4, we will see that the thermal mass correction to $m_{\text {soft }}$ only affects the thermal phase space but does not alter the coupling $g_{G}$ from its zero-temperature value, and that the thermal vertex correction is of higher order in $\alpha_{s}(T) \log \left(1 / \alpha_{s}(T)\right)$. 
The above result has an important consequence when we consider the coupling of the helicity-1/2 Goldstino to the supercurrent. Subtracting the leading divergent contributions from each operator, we find that the Goldstino coupling $g_{G}$ is essentially the zero-temperature value, whereas possible thermal corrections from gauge interactions are automatically taken into account by resummed thermal propagators and vertices inside a loop diagram when we calculate the regeneration rate. Thus the Goldstino-matter coupling $g_{G}$ ensures manifest decoupling of the Goldstino from the rest of the theory in the limit where the supersymmetry breaking in the observable sector vanishes $m_{\text {soft }} \rightarrow 0$.

Concluding this Section, for the range of supersymmetry breaking scale of phenomenological interest, we are justified in using the Goldstino equivalence theorem and effective Lagrangian Eq.(7) of the light gravitinos in the rest of this paper.

\section{REACTION RATE AT FINITE TEMPERATURE: FORMALISM}

We next formulate the particle reaction rate in a plasma, using kinetic theory [18]. Consider a heat bath consisting initially of thermal particles q's and an isolated particle species $\Phi$ slightly out of equilibrium. The heat bath of $q$ particles at temperature $T$ may be described by a density matrix

$$
\rho_{q}=\frac{1}{Z_{0}} \sum_{\{q\}} e^{-H / T}|q\rangle\langle q| .
$$

The in-state density matrix of the heat bath plus a single $\Phi$ particle is given by

$$
\rho_{\text {in }} \equiv|i n\rangle\langle i n|=a_{\Phi}^{\dagger} \rho_{q} a_{\Phi}
$$

The $\Phi$ particles then interact with other particles in the heat bath, and subsequently decay into a new thermalized out-state density matrix $\rho_{\text {out }} \equiv$ 
$|o u t\rangle\langle o u t|$. To be specific, we consider two-to-two scattering of a $\Phi$ particle on $q$ particles in the heat bath: $\Phi\left(p_{1}\right)+q\left(p_{2}\right) \rightarrow q\left(p_{3}\right)+q\left(p_{4}\right)$, whose reaction rate we denote by $\Gamma_{f}$. For simplicity, we suppress quantum numbers other than the energy and momentum of each particle. This thermal reaction differs from zero-temperature decay, in that $\Gamma_{f}$ does not specify completely the subsequent distribution of $\Phi$ particles. The inverse reaction $q\left(p_{3}\right)+a\left(p_{4}\right) \rightarrow \Phi\left(p_{1}\right)+q\left(p_{2}\right)$, whose reaction rate we denote by $\Gamma_{b}$, is also possible in general, and will create $\Phi$ particles out of the thermal bath. Unitarity dictates that $\Gamma_{f} / \Gamma_{b}=\exp (E / T)$, irrespective of any possible violation of CP. Let us denote the initial non-equilibrium distribution function of the $\Phi$ particle by $n_{i}\left(E_{1}\right)$. Subsequently, the $\Phi$ particle distribution $n_{\Phi}\left(E_{1}\right)$ evolves according to the forward and inverse reaction rates $[18,22]$

$$
\frac{d n_{\Phi}\left(t ; E_{1}\right)}{d t}=-n_{\Phi}\left(t, E_{1}\right) \Gamma_{f}+\left(1 \pm n_{\Phi}\left(t ; E_{1}\right)\right) \Gamma_{b}
$$

The \pm signs for the inverse process are for Bose-Einstein and Fermi-Dirac statistics of the $\Phi$ particle, respectively. Solving this equation, we find

$$
n_{\Phi}\left[t ; E_{1}\right]=n_{B, F}\left[E_{1}\right]+\exp \left[-\left(\Gamma_{f} \mp \Gamma_{b}\right) t\right]
$$

where $n_{B, F}[E]=1 /\left(e^{E / T} \mp 1\right)$ for boson and fermion equilibrium distribution functions, respectively. Therefore, regardless of the initial distribution, the primordial plasma approaches chemical equilibrium at sufficiently late time. As long as the starting assumption that the primordial plasma is only slightly out of equilibrium is valid, $\Gamma_{f}$ and $\Gamma_{b}$ can be calculated using the equilibrium distributions $n_{B, F}[E]$. Under this assumption, the rate of approach to equilibrium is governed by $\Gamma_{\text {tot }} \equiv \Gamma_{f} \mp \Gamma_{b}$.

It now remains to calculate the total reaction rate $\Gamma_{\text {tot }}$. The decay probability is given by

$$
\mathcal{P}=\sum_{\text {out }}\left\langle\text { out }\left|\Delta H \rho_{\text {in }} \Delta H^{\dagger}\right| \text { out }\right\rangle
$$




$$
=\frac{1}{Z_{0}} \operatorname{Tr}\left(e^{-H / T} a_{\Phi} \Delta H \Delta H^{\dagger} a_{\Phi}^{\dagger}\right)
$$

where the last formula is given in terms of retarded Green functions. Denoting the thermal phase-space volume by $d \Omega$, the reaction rate is given by

$$
\begin{aligned}
\Gamma_{\text {tot }} & =\int \frac{1}{2 E_{\Phi}} \mathcal{P} d \Omega \\
& =\frac{N_{p t}}{2 E_{1}} \iiint \frac{d^{3} \vec{p}_{2}}{(2 \pi)^{3} 2 E_{2}} \frac{d^{3} \vec{p}_{3}}{(2 \pi)^{3} 2 E_{3}} \frac{d^{3} \vec{p}_{4}}{(2 \pi)^{3} 2 E_{4}} n\left(p_{2}\right)\left(1 \pm n\left(p_{3}\right)\right)\left(1 \pm n\left(p_{4}\right)\right) \\
& \times(2 \pi)^{4} \delta^{(4)}\left(p_{1}+p_{2}-p_{3}-p_{4}\right) \operatorname{Tr}\left(a_{\Phi} \Delta H \Delta H^{\dagger} a_{\Phi}^{\dagger}\right)
\end{aligned}
$$

Here $N_{p t}=(2 s+1) N_{c} N_{f}$ denotes a factor of summing over the spin, color, flavor etc. of the particle 2 in the initial heat bath.

The thermal correlation function is evaluated with retarded boundary conditions. As such, it is naturally interpreted as a discontinuity across the physical cut relevant to the chosen process. Due to the presence of the thermal bath, there are new physical cuts present only at finite temperature. The discontinuity of the Green function is related to the imaginary part of the self-energy function of the $\Phi$ particle [22]:

$$
\Gamma_{t o t}=\Gamma_{f} \mp \Gamma_{b}=-\frac{1}{E} \operatorname{Im} \Pi[E+i \epsilon]
$$

where $\Pi[E]=\Sigma[E]$ for bosonic $\Phi$ and $\bar{\Phi}(E) \Sigma[E] \Phi(E)$ for fermionic $\Phi$. In particular, the process we are interested in is given by a cut which is present only for nonzero temperature. The real part of the self-energy function is related to the thermal correction to the mass of the particle.

\section{THERMAL REACTION RATE FOR LIGHT GRAVITINOS}

At the end of inflation, the Universe is out of chemical equilibrium, with the gravitino density depleted by the preceding inflationary period. However, scattering among particles in the primordial plasma can regenerate 
gravitinos. In this section, we calculate this gravitino regeneration rate, considering for definiteness the 2-to-2 scattering processes gluino + gluino $\rightarrow$ gluino + Goldstino or gluon + gluino $\rightarrow$ gluon + Goldstino [6]. Without taking finite-temperature effects into account, it was shown previously that $2 \rightarrow 2$ scattering was the dominant mechanism of gravitino regeneration. We are interested in the temperature range $m_{3 / 2}, m_{\text {soft }} \ll T \ll \sqrt{|F|}$. Further, we assume that the regenerated gravitinos are only slightly out of thermal equilibrium, since it is only in this situation that we can apply the equilibrium finite-temperature formalism discussed in Section 3, and that E(gravitino), $|\vec{p}|($ gravitino $) \sim \mathcal{O}(T)$, so that helicity-1/2 gravitinos are the dominant components for the scattering process. This enables us to calculate the regeneration rate using the Goldstino effective Lagrangian given in Section 2.

According to Eq.(18), the regeneration rate of Goldstinos with an energy $E$, a helicity $\lambda$ and an invariant mass-squared $s \equiv k^{\mu} k_{\mu}$ is given in terms of the discontinuity of the Goldstino self-energy diagram $\Sigma_{G}(E, \vec{k})$ depicted in Figure 3 by

$$
\Gamma_{\lambda}(E)=\frac{i}{2 E} \operatorname{disc}\left(\bar{u}_{\lambda}(k) \Sigma_{G}(E, \vec{k}) u_{\lambda}(k)\right)
$$

where the Goldstino spinor wave function is denoted by $u(k)$, and satisfies

$$
\left(\gamma^{0} E-\vec{\gamma} \cdot \vec{k}\right) u(k)=0 ; \quad \bar{u}(k) u(k)=2 \sqrt{s}
$$

We recall that the decay probability of the Goldstino is related to the regeneration probability, once the discontinuity in Eq.(19) is taken across an appropriate kinematical cut in the complex $E$ or $s$ plane. However, the stimulated-emission factor $n_{F}(E)$ relevant for the Goldstino decay rate should be replaced by the Pauli-blocking factor $1-n_{F}(E)$ relevant for the regeneration rate. Summing over the Goldstino helicity states $\lambda$, we obtain the total regeneration rate 


$$
\begin{aligned}
\Gamma_{G}(E) & =\frac{i}{2 E}\left(1-n_{F}(E)\right) \operatorname{disc} \sum_{\lambda} \bar{u}_{\lambda}(k) \Sigma_{G}(E, \vec{k}) u_{\lambda}(k) \\
& =-\frac{1}{E}\left(1-n_{F}(E)\right) \operatorname{Tr}\left[(\not k+\sqrt{s}) \operatorname{Im} \Sigma_{G}(E+i \epsilon, \vec{k})\right] .
\end{aligned}
$$

In what follows, we consider near-on-shell Goldstinos and set $s=0$. The Goldstino self-energy $\Sigma_{G}(E, \vec{k})$ is calculated using the effective Lagrangian of Section 2. We emphasize again the important conclusion drawn in Section 2 that we can use the zero-temperature Goldstino-matter coupling $g_{G}=m_{\text {soft }}^{2} /|F|$ when evaluating $\Sigma_{G}(E, \vec{k})$. Finite-temperature corrections to $g_{G}$ will be taken automatically into account by the resummed propagators and vertices. The imaginary part of $\Sigma$ can be expressed as a sum of integrals over the phase space of initial and final heat-bath states weighted by statistical distributions. The integrands are squares of a mplitudes of the form (Goldstino) $+2 \leftrightarrow 3+4$, where 2,3,4 are particles in the plasma heat bath, which we have taken as gluinos and gluons.

In thermal field theory, the Goldstino self-energy diagram may be calculated using effective thermal vertices and propagators. The skeleton diagram given in Fig. 3 is then evaluated in Minkowski space as $\left(k^{\mu} \equiv(E, \vec{k})\right)$

$$
\Sigma_{G}(E, \vec{k})=\int \frac{d^{4} q}{(2 \pi)^{4}} q^{\mu} \Gamma_{\mu \alpha}(q) \Pi^{\alpha \beta}(q) S_{\tilde{g}}(k-q) \Gamma_{\beta \nu}(q) q^{\nu}
$$

Here $\Pi^{\mu \nu}, S_{\tilde{g}}$ and $\Gamma_{\mu \nu}$ are effective thermal gluon and gluino propagators and the effective thermal vertex for gluon-gluino-gluino coupling, respectively, in which naive perturbative diagrams are resummed. It is well known that in hot gauge theories such a resummation is necessary to take screening effects into account correctly. So far, in gauge theories a consistent resummation method has been developed only using the imaginary-time formalism of thermal field theory, mainly by Braaten and Pisarski [17]. The above regeneration rate formula Eq.(21) has the advantage that the Goldstino self-energy $\Sigma_{G}(E, \vec{k})$ can be calculated straightforwardly using the same imaginary-time formalism. 
We now apply the Braaten-Pisarski method to calculate the Goldstino selfenergy in a resummed perturbative expansion.

From the structure of $\Sigma_{G}(E, \vec{k})$ in Eq.(22), it is easy to see that the regeneration rate falls off rapidly for a large momentum transfer to the internal gluon line, i.e., the $\vec{q}$ integrand has $\sim 1 /|\vec{q}|$. This gives logarithmic divergences at small and large $|\vec{q}|$. Therefore it is convenient to introduce some arbitrary scale $\bar{q}$ such that $g T \ll \bar{q} \ll T$ and divide the $|\vec{q}|$ kinematics into soft $g T \lesssim|\vec{q}| \ll \bar{q}$ and hard $\bar{q}<|\vec{q}| \lesssim T$ regimes. Since $\bar{q}$ is an arbitrary scale, any physical quantity should be independent of it.

For the soft regime, the energy and momentum through the gluino internal line are hard, namely $E-q^{0},|\vec{k}-\vec{q}| \sim \mathcal{O}(T)$, so a bare gluino propagator suffices. We only need to retain an effective gluon propagator which resums the bubble diagrams. As we have argued earlier, we also assume that the external Goldstino has energy and momentum of order $\sim T$. In this case a bare Goldstino-matter coupling $g_{G}=m_{\text {soft }}^{2} /|F|$ suffices. Only at higher orders or for Goldstinos whose energy and momentum are less than $\mathcal{O}(T)$ it is necessary take into account a different effective Goldstino-matter coupling. We will discuss this later in this Section. For the hard regime, both the gluon and gluino internal lines are hard. Thus this contribution may be calculated using bare vertices and propagators. In the following calculation, we take the leading logarithmic approximation, for which the total reaction rate may be obtained just from the calculation in the soft regime and the hard contribution is automatically taken into account by the requirement that the final result should be independent of the arbitrarily introduced scale $\bar{q}$.

To cure the logarithmic divergence in the soft regime, it is necessary to resum the bubble diagrams of the gluon self-energy following the BraatenPisarski method. As we will see, the resummation turns the infrared-divergent contribution into an infrared-finite one. It is known that the Braaten-Pisarski 
method is gauge-independent for any physical observable such as the regeneration rate. Therefore we are free to choose any convenient gauge, and will choose Coulomb gauge in the subsequent calculations. In this gauge, denoting $q^{\mu} \equiv\left(q_{0}, \vec{q}\right), q \equiv|\vec{q}|$, the nonzero components of the effective gluon propagator $\Pi^{\mu \nu}$ after the resummation are known [23]

$$
\begin{aligned}
& \Pi^{00}(\omega, q)=\Delta_{L}(\omega, q), \\
& \Pi^{i j}(\omega, q)=\left(\delta^{i j}-\frac{q^{i} q^{j}}{q^{2}}\right) \Delta_{T}(\omega, q) .
\end{aligned}
$$

Here

$$
\begin{aligned}
& \Delta_{L}(\omega, q) \equiv\left[q^{2}-\frac{3}{2} m_{g}^{2}\left\{\frac{\omega}{q} \ln \left(\frac{\omega+q}{\omega-q}\right)-2\right\}\right]^{-1} \\
& \Delta_{T}(\omega, q) \equiv\left[\omega^{2}-q^{2}+\frac{3}{2} m_{g}^{2}\left\{\frac{\omega}{2}\left(\frac{\omega^{2}}{q^{2}}-1\right) \ln \left(\frac{\omega+q}{\omega-q}\right)-\left(\frac{\omega}{q}\right)^{2}\right\}\right]^{-1}
\end{aligned}
$$

in which $m_{g}^{2}(T) \equiv \frac{8 \pi}{3}\left(1+n_{f} / 6\right) \alpha_{s}(T) T^{2}\left(n_{f}=\right.$ number of light quark flavors in the fundamental representation) is derived for the supersymmetric particle content in the high-temperature limit $\omega, q \ll T$ and may be interpreted as an effective gluon mass generated by interactions with the primordial plasma.

Using this, the self-energy $\Sigma_{G}(E, \vec{k})$ Eq.(22) simplifies to

$$
\Sigma_{G}(E, \vec{k})=i\left|\frac{m_{\text {soft }}}{8 F}\right|^{2} \int \frac{d^{4} q}{(2 \pi)^{4}} q^{\mu} \gamma_{\mu \alpha} \frac{\Pi^{\alpha \beta}(q)}{\not k-\not q-m_{\text {soft }}} \gamma_{\beta \nu} q^{\nu} .
$$

Next we take the gamma matrix trace of the product of $\Sigma_{G}(E, \vec{k})$ with the Goldstino projection operator given in Eq.(21):

$$
\operatorname{Tr}\left[\not k \Sigma_{G}(E, \vec{k})\right]=-\left|\frac{m_{\text {soft }}}{8 F}\right|^{2} \sum_{q_{0}} \int \frac{d^{3} \vec{q}}{(2 \pi)^{3}} \frac{A \Delta_{L}\left(q_{0}, q\right)+B \Delta_{T}\left(q_{0}, q\right)}{\left(E-q_{0}\right)^{2}-(\vec{k}-\vec{q})^{2}-m_{\text {soft }}^{2}}
$$

where $A$ and $B$ are calculated using the on-shell condition $E^{2}-\vec{k}^{2}=0$ as

$$
\begin{aligned}
A=4[ & \left.-2(\vec{k} \cdot \vec{q})^{2}+(\vec{k} \cdot \vec{q}) q^{2}+2 q^{2} E^{2}-q_{0} q^{2} E\right], \\
B=8[ & -(\vec{k} \cdot \vec{q})\left(3 \vec{k} \cdot \vec{q}-q^{2}\right)+E^{2} q^{2}+q_{0}\left\{4 \vec{k} \cdot \vec{q}-q^{2}\right\} E \\
& \left.+q_{0}^{2}\left\{-3 E^{2}-(\vec{k} \cdot \vec{q})+(\vec{k} \cdot \vec{q})^{2} / q^{2}\right\}+q_{0}^{3} E\right] .
\end{aligned}
$$


A possible soft gluino-mass dependence in the numerator drops out after the trace over the gamma matrices is taken. To simplify further calculations, we have also ignored the soft gluino mass in the gluino propagator. Next we sum over the discrete energies $q_{0}=2 \pi i n T$. This is most easily done using the spectral representation for the gluino and the effective gluon propagators. For the gluino propagator

$$
\begin{aligned}
& \frac{1}{\left(E-q_{0}\right)^{2}-(\vec{k}-\vec{q})^{2}}=-\frac{1}{\pi} \operatorname{Im}\left[\frac{1}{\left(E-q_{0}+i \epsilon\right)^{2}-(\vec{k}-\vec{q})^{2}}\right] \\
= & -\frac{1}{2|\vec{k}-\vec{q}|} \int_{0}^{\frac{1}{T}} d \tau_{2} e^{\left(E-q_{0}\right) \tau_{2}}\left\{\left(1-n_{F}(|\vec{k}-\vec{q}|)\right) e^{-|\vec{k}-\vec{q}| \tau_{2}}-n_{F}(|\vec{k}-\vec{q}|) e^{+|\vec{k}-\vec{q}| \tau_{2}}\right\} .
\end{aligned}
$$

For the effective gluon propagator [24]

$$
\begin{aligned}
& \Delta_{L}\left(q_{0}, q\right)=-\frac{1}{\pi} \operatorname{Im} \Delta_{L}\left(q_{0}+i \epsilon, q\right)=-\int_{0}^{\frac{1}{T}} d \tau_{1} e^{q_{0} \tau_{1}} \int_{-\infty}^{\infty} d \omega \rho_{L}(w, q) e^{-\omega \tau_{1}}\left[1+n_{B}(\omega)\right], \\
& \Delta_{T}\left(q_{0}, q\right)=-\frac{1}{\pi} \operatorname{Im} \Delta_{T}\left(q_{0}+i \epsilon, q\right)=-\int_{0}^{\frac{1}{T}} d \tau_{1} e^{q_{0} \tau_{1}} \int_{-\infty}^{\infty} d \omega \rho_{T}(w, q) e^{-\omega \tau_{1}}\left[1+n_{B}(\omega)\right] .
\end{aligned}
$$

They have nonzero support in the complex $\omega$ plane:

$$
\begin{aligned}
\rho_{L}(\omega, q) & =\frac{3}{2} m_{g}^{2} \frac{\omega}{q}\left|\Delta_{L}(\omega+i \epsilon, q)\right|^{2} \\
& =\frac{3}{2} m_{g}^{2} \frac{\omega}{q}\left[\frac{9 \pi^{2}}{4} m_{g}^{4}\left(\frac{\omega}{q}\right)^{2}+\left\{q^{2}+3 m_{g}^{2}-\frac{3}{2} m_{g}^{2} \frac{\omega}{q} \log \left(\frac{q+\omega}{q-\omega}\right)\right\}^{2}\right]^{-1}, \\
\rho_{T}(\omega, q) & =\frac{3}{4} m_{g}^{2}\left(\frac{\omega}{q}-\left(\frac{\omega}{q}\right)^{3}\right)\left|\Delta_{T}(\omega+i \epsilon, q)\right|^{2} \\
& =\frac{3}{4} m_{g}^{2}\left(\frac{\omega}{q}-\left(\frac{\omega}{q}\right)^{3}\right)\left[\frac{9 \pi^{2}}{16} m_{g}^{4}\left(\frac{q}{\omega}-\frac{\omega}{q}\right)^{2}\right. \\
& \left.+\left\{q^{2}-\omega^{2}+\frac{3}{2} m_{g}^{2}\left(\frac{\omega}{q}\right)^{2}\left(1+\frac{1}{2}\left(\frac{q}{\omega}-\frac{\omega}{q}\right) \log \left(\frac{q+\omega}{q-\omega}\right)\right)\right\}^{2}\right]^{-1}
\end{aligned}
$$

for $-q<\omega<+q$ in addition to the plasmon and the transverse gluon delta function poles at $\omega= \pm \omega_{L}(q), \pm \omega_{T}(q)$ respectively.

After rewriting the propagators in terms of these spectral representations, it is easy to see that the sum over discrete $q_{0}$ yields expressions of the form

$$
\sum_{q_{0}} q_{0}^{n} e^{q_{0}\left(\tau_{1}-\tau_{2}\right)}=\left(\frac{\partial}{\partial \tau_{1}}\right)^{n} \delta\left(\tau_{1}-\tau_{2}\right)
$$

for $n=0,1,2,3$, relevant for $A$ and $B$ in Eq.(27). Integration over $\tau_{1,2}$ then yields a factor of the form 


$$
-\frac{1}{E \mp|\vec{k}-\vec{q}|-\omega}\left(e^{(\mp|\vec{k}-\vec{q}|-\omega) / T}+1\right)
$$

in which we have used the fact that $E=2 \pi i(n+1 / 2) T$ for the Goldstino. After all these steps are taken, the overall dependence of $\operatorname{Tr}\left[\not k \operatorname{Im} \Sigma_{G}(E, \vec{k})\right]$ resides only in the energy denominator in Eq.(32):

$$
\operatorname{Im} \frac{1}{(E+i \epsilon) \mp|\vec{k}-\vec{q}|-\omega}=-\pi \delta(E \mp|\vec{k}-\vec{q}|-\omega)
$$

Therefore, we finally obtain for the imaginary part of the trace in Eq.(26):

$$
\begin{aligned}
\operatorname{Tr} & {\left[\not k \operatorname{Im} \Sigma_{G}(E, \vec{k})\right] } \\
& =-\pi\left|\frac{m_{\text {soft }}}{8 F}\right|^{2} \frac{1}{\left(1-n_{F}(E)\right)} \\
& \times \int \frac{d^{3} \vec{q}}{(2 \pi)^{3}} \int_{-\infty}^{\infty} d \omega\left[\mathcal{A}\left(\rho_{L}(\omega, q)+\mathcal{B} \rho_{T}(\omega, q)\right]\right. \\
& \times \frac{1}{2 \mathcal{E}}\left(1+n_{B}(\omega)\right)\left[\left(1-n_{F}(\mathcal{E})\right) \delta(E-\mathcal{E}-\omega)-n_{F}(\mathcal{E}) \delta(E+\mathcal{E}-\omega)\right]
\end{aligned}
$$

in which $\mathcal{E} \equiv|\vec{k}-\vec{q}|$ and $\mathcal{A}, \mathcal{B}$ are given by $A, B$ in Eq.(27) with $q_{0}$ replaced by $\omega$.

Recall that $E,|\vec{k}-\vec{q}|,|\vec{k}| \approx \mathcal{O}(T) \gg q \approx \mathcal{O}(g T)>|\omega|$. Because of this, the second delta function in Eq.(34) does not contribute at all. In addition, $e^{-\omega / T}\left(1+n_{B}(\omega)\right) \approx T / \omega$ and $|\vec{k}-\vec{q}| \approx k+\vec{k} \cdot \vec{q} / k$. Then the angular $\vec{q}$ integration can be done straightforwardly with the first delta function in Eq.(34) and gives rise to a restriction to $|\omega|<q$, unique to the finite-temperature regeneration process. After these manipulations the reaction rate is given by

$$
\begin{aligned}
\Gamma_{G}(E) & =\frac{2}{\pi} T\left|\frac{m_{\text {soft }}}{8 F}\right|^{2} \\
& \times \int_{0}^{\bar{q}} q^{3} d q \int_{-q}^{+q} \frac{d \omega}{\omega}\left\{1-\frac{\omega^{2}}{q^{2}}\right\}\left[\rho_{L}(\omega, q)+\left(1-\frac{\omega^{2}}{q^{2}}\right) \rho_{T}(\omega, q)\right] .
\end{aligned}
$$

After appropriate rescaling and changing the order of integration, we have done the $q$ integral analytically and the $\omega$ integral numerically. The result is given by

$$
\Gamma_{G}=\frac{3}{\pi} T m_{g}^{2}(T)\left|\frac{m_{\text {soft }}}{8 F}\right|^{2}\left[\log \left(\frac{\bar{q}^{2}}{m_{g}^{2}}\right)-1.38+\cdots\right]
$$


At small $\alpha_{s}(T)$, which is relevant to our case, the first term is the dominant, leading-logarithmic contribution. The second term is a subleadinglogarithmic contribution. As explained earlier, the dependence on the arbitrarily-introduced scale $\bar{q}$ is a theoretical artifact. Once we add the contribution from hard regime, this dependence should disappear [25]. Therefore, without an explicit calculation for the hard regime, the regeneration rate in the leading-logarithmic approximation can be obtained from Eq.(36).

In the above evaluation we have used the bare Goldstino-matter coupling constant $g_{G}=m_{\text {soft }}^{2} /|F|$. According to power counting, this is justified since the Goldstino and the gluino have energy and momentum of order $\mathcal{O}(T)$. The thermal-loop correction to the Goldstino vertex may be evaluated by calculating the Figure $2(a)$ and (b) in which the blob consists of resummed, effective internal propagators on the gluon-gluino or chiral matter boson-fermion lines. Let us consider Figure 2 (a). By power counting, the vertex correction is quadratically divergent for small $q$ along the internal gluon line. Therefore it is necessary to use the effective gluon propagator Eq.(23) as well as effective thermal masses for the internal particles. These resummations then soften the quadratic infrared divergence of the integrand into a logarithmic one. The result is that the Goldstino-matter coupling receives a correction of the form

$$
g_{G}(T)=g_{G}\left(1+\alpha_{s}(T) \log \left(1 / \alpha_{s}(T)\right)+\cdots\right)
$$

The ellipses denote subleading $\mathcal{O}\left(\alpha_{s}(T)\right)$ or higher-order corrections.

Combining Eq.(37) with the correction Eq.(11) from the Goldstino dynamics, we find that the gravitino regeneration rate is given by

$$
\begin{aligned}
\Gamma_{G} & =\frac{1}{16}\left(1+\frac{n_{f}}{6}\right)\left|\frac{m_{\text {soft }}}{F}\right|^{2} T^{3} \alpha_{s}(T) \log \left(1 / \alpha_{s}(T)\right) \\
& \times\left[1+\alpha_{s}(T) \log \left(1 / \alpha_{s}(T)\right)+\frac{T^{2}}{|F|}+\cdots\right] .
\end{aligned}
$$


We have suppressed model-dependent numerical factors of order unity in each of the correction terms.

Eq.(38) is the main result of this paper. It shows that the regeneration rate calculated in the high-temperature limit and in the leading-logarithmic approximation is proportional to $T^{3}$. This temperature dependence agrees qualitatively with the one obtained from the zero-temperature calculation [6]. When summed over the minimal supersymmetric standard model particle content, the numerical prefactor in Eq.(38) is $\sim 0.291$. In the earlier calculation of [6] the Boltzmann equation for the gravitino number density $n_{3 / 2}$ was used to deduce the regeneration rate

$$
\Gamma_{3 / 2} \approx \frac{1}{n_{\text {rad }}} \frac{d}{d t} n_{3 / 2}(T) \approx \Sigma_{\text {tot }} n_{\text {rad }}(T) .
$$

where the expansion rate of the Universe and possible contributions from heavy-particle decay are neglected in Eq.(39), and the zero-temperature total cross section is denoted by $\Sigma_{t o t}$. From the result of [6] it is easy to check that the leading-logarithmic contribution is essentially the same as ours in Eq.(38), except for a slightly different numerical prefactor $\sim 0.250$ for the minimal supersymmetric model particle content (after correcting discrepancies by factors of 2 in the Goldstino effective Lagrangian used in [6]). The finite-temperature calculation, however, was essential in our case to soften infrared divergences and yield the finite result of Eq.(38) in the leading-logarithmic approximation. In contrast, in [6] the infrared divergence was cut off in an ad hoc manner. Furthermore the factor $\log \left(1 / \alpha_{s}(T)\right)$ implies that the thermal $Q C D$ effects mainly originate over the range from the extreme soft $q \sim g T$ to the extreme hard $q \sim T$ regime. 


\section{COSMOLOGICAL IMPLICATIONS}

In the absence of inflation, there are clear limits on the gravitino mass [1]. A stable gravitino (e.g., if the gravitino were the LSP) would contribute to the present overall mass density an amount

$$
\rho_{3 / 2}=m_{3 / 2} n_{3 / 2} \sim O\left(10^{-2}\right) m_{3 / 2} n_{\gamma}
$$

where $n_{\gamma}$ is the present density of photons. In order that $\Omega_{3 / 2} h^{2} \leq \frac{1}{4}$, where $h$ is the present Hubble expansion rate of the Universe in units of $100 \mathrm{kms}^{-1} \mathrm{Mpc}^{-1}$, one requires $m_{3 / 2}<O(1) \mathrm{keV}$. More massive gravitinos must be unstable. Because the gravitino decay rate is suppressed, $\Gamma_{3 / 2} \sim m_{3 / 2}^{3} / M_{P}^{2}$, gravitinos come to dominate the energy density of the Universe prior to their decay, when $T_{D} \sim m_{3 / 2}^{5 / 3} / Y^{1 / 3} M_{P}^{2 / 3}$ (see, e.g., [4]), where $Y=n_{3 / 2} / n_{\gamma} \sim 10^{-2}$ in the absence of inflation. In cosmologies with inflation, $Y$ may be much smaller, but in this case gravitinos may never dominate the energy density of the Universe. Subsequent to gravitino decay, the Universe "reheats" to a temperature, $T_{R} \sim m_{3 / 2}^{3 / 2} / M_{P}^{1 / 2}$ and, for the Universe to recover prior to nucleosynthesis, we must have $m_{3 / 2} \gtrsim 20 \mathrm{TeV}$. However, even in this case one must still be concerned about the dilution of the baryon-to-entropy ratio [2], which would be by a factor $\Delta=\left(T_{R} / T_{D}\right)^{3} \sim Y\left(M_{P} / m_{3 / 2}\right)^{1 / 2}$. Dilution may not be a problem if the baryon-to-entropy ratio is initially large.

Inflation modifies the above constraints on the gravitino mass [2]. During inflation, the abundance of gravitinos relative to photons is dramatically reduced, as is the abundance of many other unwanted relics. The problem with gravitinos, however, is that they are regenerated as the Universe rethermalizes after inflation. If gravitinos satisfy the noninflationary bounds, then their reproduction after inflation is never a problem. For gravitinos with mass of order $100 \mathrm{GeV}$, dilution without over-regeneration will also solve the 
problem, but there are several factors one must contend with in order to be cosmologically safe. Gravitino decay products can also upset the successful predictions of Big Bang nucleosynthesis [6], and decays into LSPS (if R-parity is conserved) can also yield too large a mass density in the now-decoupled LSPS [5].

Let us consider first the constraints imposed by regeneration. Using the rate of gravitino regeneration given by $\mathrm{Eq}$. (38), the abundance of gravitinos produced after inflation will be $Y \sim \Gamma / H, H \simeq \sqrt{N} T^{2} / M_{P}$, where $N$ is the number of degrees of freedom. The most stringent of the Big Bang nucleosynthesis constraints comes from the photoproduction of deuterium and ${ }^{3} \mathrm{He}[7]$ and yields the limit

$$
Y<3 \times 10^{-14}\left(\frac{100 G e V}{m_{3 / 2}}\right)
$$

coresponding to a limit on the reheating temperature after inflation of

$$
T_{R}<2.5 \times 10^{8}\left(\frac{100 G e V}{m_{3 / 2}}\right) G e V
$$

A slightly stronger bound (by an order of magnitude in $T_{R}$ ) was found in [11]. It is evident that if the goldstino regeneration rate were larger by a factor of $T^{2} / m_{3 / 2}^{2}$, then the left side of (42) would become $T_{R}^{3} / m_{3 / 2}^{2}$, and the upper limit on $T_{R}$ would be about $10^{4} \mathrm{GeV}\left(\mathrm{m}_{3 / 2} / 100 \mathrm{GeV}\right)$.

Given the severity of even the previous limits, e.g., eq. (42), it is important that this limit be put into perspective with what we expect from typical inflationary models, and with what we require from the point of view of Big Bang baryogenesis. Inflationary models [26] can in principle be described by a single dimensionful parameter $\mu$ which is fixed $[4,27,28]$ by the magnitude of the observed microwave background fluctuations [29]: $\mu^{2} / M_{P}^{2} \simeq$ few $\times 10^{-8}$. Because it is assumed that the inflaton potential is of magnitude $\mu^{4}$, the inflaton mass $m_{\eta}$, the duration of inflation described by the number of efoldings $H \tau$, and the reheating temperature $T_{R}$ are all given by $\mu$ : 


$$
\begin{aligned}
& m_{\eta} \sim \mu^{2} / M_{P} \sim f e w \times 10^{11} \mathrm{GeV} \\
& H \tau \sim 8 \pi\left(M_{P} / \mu\right)^{2} \sim 10^{9} \\
& T_{R} \sim \mu^{3} / M_{P}^{2} \sim 10^{8} \mathrm{GeV}
\end{aligned}
$$

However, in [27] it was noted that in fact the Universe is not immediately thermalized subsequent to inflaton decays, and the process of thermalization actually leads to a smaller reheating temperature,

$$
T_{R} \sim \alpha^{2} \mu^{3} / M_{P}^{2} \sim 10^{5} G e V
$$

where $\alpha^{2} \sim 10^{-3}$ characterizes the strength of the interactions leading to thermalization. This low reheating temperature is certainly safe with regards to the gravitino limit (41) discussed above. Even if there is a more efficient reheating mechanism leading to a higher reheating temperature $T_{R}$, or if there is significant non-thermal gravitino production before thermalization [30], we do not expect the equivalent $T_{R}$ to lie above (42), which is also compatible with our gravitino bound (41).

It is sometimes asserted that a low reheating temperature, (one compatible with the bounds from gravitino regeneration) is incompatible with baryogenesis above the weak scale. However, what is important is not the value of $T_{R}$, but rather the inflaton mass $m_{\eta}$. With $m_{\eta}>10^{11} \mathrm{GeV}$, there are several possibilities for baryogenesis besides electroweak baryogenesis [31]. Even the simple out-of-equilibrium decay of GUT Higgs bosons with masses $m_{H}<m_{\eta}$ can generate a sizeable asymmetry. As long as inflaton decay can produce these Higgses, they will be present and out of equilibrium at a temperature $T \ll m_{H}[4,32]$. In this context, the Affleck-Dine mechanism [33], involving flat sfermion directions of the scalar potential also works quite efficiently [27]. There is also the interesting mechanism proposed by Fukugita and Yanagida [34], which generates a lepton asymmetry by the decay of a heavy right-handed 
neutrino. Nonperturbative electroweak interactions associated with sphaleron transitions then reprocess this lepton asymmetry into a baryon asymmetry. All that is required is that the mass of the right-handed Majorana neutrino be less than the inflaton mass [28]. This provides an upper bound on the right-handed neutrino mass, of about $10^{12} \mathrm{GeV}$, which in turn implies that left-handed neutrinos cannot be arbitrarily light, and suggests that they are likely to be in the range of astrophysical interest, as was discussed explicitly in the context of flipped SU(5) in [35]. Clearly there is no difficulty in generating a baryon asymmetry while at the same time satisfying the constraints imposed by gravitino regeneration.

Note that if the goldstino production rate was as large as claimed in [10], then gravitino masses less than a few $\mathrm{TeV}[7]$ would be excluded, as the nucleosynthesis contraints would imply that $T_{R}<10^{4} \mathrm{GeV}$. Heavier gravitinos would not be subject to the nucleosynthesis bound, as in this case gravitinos would have decayed before nucleosynthesis, and only the weaker bounds, which and are consistent with $T_{R} \sim 10^{5} \mathrm{GeV}$, apply.

Finally, we would like to touch briefly upon a related problem, namely that excess entropy production by the scalar fields often thought to be associated with local supersymmetry breaking, namely the Polonyi problem [36]. The Polonyi problem is in general more dangerous than the gravitino problem, as it is not resolved by inflation. During inflation, the scalars are in general driven to field values which differ from the global minimum after inflation. There is, however the possibility that these scalars are in fact quite massive [37]. In no-scale supergravity models, the masses of these scalars (and the gravitino as well) are not determined at the tree level, despite the local breaking of supersymmetry. If these masses are large, as in [37], there is no longer a problem with either the scalars or the gravitino [38]. So long as the masses of the scalars are more than $\sim 10^{-8} M_{P}$, there is no appreciable 
entropy production [39], and if the scalar masses are larger than $\sim 10^{-12} M_{P}$ they never dominate the energy density [27], and are therefore acceptable. Below this mass, the scalars do dominate, and their decays to LSP's can lead to an excessive value for the present mass density [40].

\section{CONCLUSION}

In this paper, we have investigated the light gravitino regeneration rate in the early Universe with particular emphasis on finite-temperature effects. We first found that thermal corrections to the gravitino mass are negligibly small. We thus conclude that the helicity-1/2 components of the gravitino dominate reaction processes in the primordial plasma. Using Weldon's discontinuity rule [22], we then related the Goldstino regeneration rate to the discontinuity of the Goldstino self energy across the appropriate kinematical cut in the complex energy plane. To evaluate the Goldstino self energy, we used the low-energy effective Lagrangian for helicity-1/2 components. This is justified by the fact that the thermal correction to the gravitino mass is completely negligible, and the temperature is in the range $m_{\text {soft }} \ll T \ll \sqrt{F}$.

We have also examined thermal correction to the Goldstino-matter coupling $g_{G}=m_{\text {soft }}^{2} /|F|$. Naively one may expect that $m_{\text {soft }}^{2}$ is replaced by thermal mass $\sim \alpha_{s}(T) T^{2}$. By careful study, we have found that no such large correction arises. Because all the thermal corrections are to be calculated using the zero temperature $g_{G}=m_{\text {soft }}^{2} /|F|$, decoupling of the supersymmetry breaking $m_{\text {soft }} \rightarrow 0$ must be regular even at high temperature. We have shown this through detailed calculations. Both the Goldstino dynamics and the $Q C D$ interactions give rise to corrections of order $T^{2} /|F|$ and $\alpha_{s}(T) \log \left(1 / \alpha_{s}(T)\right)$ respectively. Numerically they are negligible since $M_{W} \sim m_{\text {soft }} \ll T \ll \sqrt{|F|}$. 
At least up to the order we have calculated, the Braaten-Pisarski method we have utilized does not exhibit manifest supersymmetry since powercounting and kinematical consideration dictates that an effective gluon propagator is essential although a bare gluino propagator is sufficient. In the effective Goldstino Lagrangian, the local supersymmetry of the underlying supergravity theory is realized nonlinearly as a global supersymmetry among Goldstino, chiral matter and gauge supermultiplets. A full order calculation may retain the Ward identity of the global supersymmetry. However, even without appealing to the supersymmetry Ward identity, our final result has shown that an earlier estimate of the regeneration rate based on zero-temperature calculations remains qualitatively correct, up to leading logarithmic factor, which arises only in the full resummed finite-temperature perturbation method. In particular, at leading logarithmic approximation, we have found that the regeneration rate was proportional to $T^{3}$. This is in disagreement with Fischler's estimate that the rate goes as $T^{5}$ at high temperature.

We also have discussed the implications of our results for baryogenesis, and argued that they are compatible with many plausible scenarios including the Affeck-Dine mechanism and right-handed neutrino decay.

As we were finishing our work, we received a related preprint by Leigh and Rattazzi [41]. They restricted themselves to supergravity theories with a hidden sector in which the supersymmetry is transmitted to the observable sector at high energy, near the Planck scale. Their argument uses the supersymmetry Ward identity to justify naive dimensional analysis, whereas our results are more explicit. Whilst the supersymmetry Ward identity may play some role, we have not explicitly relied on it in our actual calculations. Nevertheless, our conclusion agrees with theirs where we overlap. 


\section{ACKNOWLEDGEMENTS}

SJR thanks Andrei Linde for several helpful discussions, and the hospitality of the Theory Group at CERN where this work was initiated. This work was supported in part by U.S. NSF-KOSEF Bilateral Grant(SJR), KRF Nondirected Research Grant 81500-1341 (SJR), KOSEF Purpose-Oriented Grant 94-1400-04-01-3 and SRC Program (SJR) and Ministry of Education BSRI-94-2418 (SJR); DOE grant DE-FG05-91-ER-40633DOE (DVN); DOE grant DE-FG02-94ER40823 (KAO). 


\section{REFERENCES}

[1] S. Weinberg, Phys. Rev. Lett. 48 (1982) 1303.

[2] J. Ellis, A.D. Linde and D.V. Nanopoulos, Phys. Lett. 118 (1982) 59;

L.M. Krauss, Nucl. Phys. B227 (1983) 556.

[3] H. Pagels and J. Primack, Phys. Rev. Lett. 48 (1982) 223;

M.Y. Khlopov and A.D. Linde, Phys. Lett. B138 (1984) 265.

[4] D.V. Nanopoulos, K.A. Olive and M. Srednicki, Phys. Lett. $127 B$ (1983) 30.

[5] J. Ellis, J. Hagelin, D.V. Nanopoulos, K.A. Olive and M. Srednicki, Nucl. Phys. $B 238$ (1984) 453.

[6] J. Ellis, J.E. Kim and D.V. Nanopoulos, Phys. Lett. B145 (1984) 181.

[7] J. Ellis, D.V. Nanopoulos and S. Sarkar, Nucl. Phys. B259 (1985) 175;

R. Juszkiewicz, J. Silk and A. Stebbins, Phys. Lett. $158 B$ (1985) 463;

D. Lindley, Phys. Lett. B171 (1986) 235;

M. Kawasaki and K. Sato, Phys. Lett. B189 (1987) 23.

[8] V.S. Berezinskii, Phys. Lett. B261 (1991) 71.

[9] T. Moroi, H. Murayama and M. Yamaguchi, Phys. Lett. B303 (1993) 289.

[10] W. Fischler, Phys. Lett. B332 (1994) 277.

[11] M. Kawasaki and T. Moroi, Tohoku preprint hep-ph/9403364 (1994).

[12] T. Moroi, Ph.D. thesis, hep-ph/9503210 (1995).

[13] D. Boyanovsky, Phys. Rev. D29 (1984) 743;

H. Aoyama and D. Boyanovsky, Phys. Rev. D30 (1984) 1356;

H. Matsumoto, M. Nakahara, Y. Nakano and H. Umezawa, Physica 15D (1985) 163; 
H. Aoyama, Phys. Lett. B171 (1986) 420.

[14] A. Das and M. Kaku, Phys. Rev. D18 (1978) 4540.

[15] L. Girardello, M.T. Grisaru and P. Salomonson, Nucl. Phys. B178 (1981) 331.

[16] A.L. Fetter and J.D. Walecka, Quantum Theory of Many-Particle Systems, McGraw-Hill Pub. Co., New York, 1971.

[17] R.D. Pisarski, Phys. Rev. Lett. 63 (1989) 1129;

E. Braaten and R.D. Pisarski, Phys. Rev. Lett. 64 (1990) 1338;

E. Braaten, R.D. Pisarski and T.C. Yuan, Phys. Rev. Lett. 64 (1990) 2242;

E. Braaten and R.D. Pisarski, Nucl. Phys. B337 (1990) 569;

E. Braaten and R.D. Pisarski, Phys. Rev. D45 (1992) R1827.

[18] E.M. Lifshitz and L.P. Pitaevskii, Physical Kinetics, Pergamon Press, Oxford, 1993; F. Reif, Fundamentals of Statistical and Thermal Physics, McGraw-Hill Pub. Co., New York, 1965.

[19] P. Fayet, Phys. Lett. B69 (1977) 489; ibid. B7o (1977) 461; ibid. B117 (1982) 460; ibid. B175 (1986) 471.

[20] For a review see: A.B. Lahanas and D.V. Nanopoulos, Phys. Rep. 145 (1987) 1.

[21] V.L. Eletsky and I.I. Kogan, Nucl. Phys. (Proc. Suppl.) 39B,C (1995) 467.

[22] H.A. Weldon, Phys. Rev. D28 (1983) 2007.

[23] V.P. Silin, Sov. Phys. JETP, 11 (1960) 1136;

O. Kalashnikov and V.V. Klimov, Sov. J. Nucl. Phys. 31 (1980) 699;

V.V. Klimov, Sov. Phys. JETP 55 (1982) 199;

H.A. Weldon, Phys. Rev. D26 (1982) 1394.

[24] R.D. Pisarski, Physica A158 (1989) 146. 
[25] E. Braaten and T.C. Yuan, Phys. Rev. Lett. 66 (1991) 2183.

[26] For reviews, see: A.D. Linde, Particle Physics And Inflationary Cosmology, Harwood (1990);

K.A. Olive, Phys.Rep. C190(1990) 307.

[27] J. Ellis, K. Enqvist, D.V. Nanopoulos and K.A. Olive, Phys. Lett. B191(1987) 343.

[28] B. Campbell, S. Davidson, and K.A. Olive, Nucl. Phys. B399 (1993) 111.

[29] G.F. Smoot et al. Ap.J 396 (1992) L1;

E.L. Wright et al. Ap.J. 396 (1992) L13 (1993) 111.

[30] L. Kofman, A. Linde and A. Starobinsky, Phys. Rev. Lett. 73 (1994) 3195;

D. Boyanovsky et al., Univ. of Pittsburg preprint PITT-09-95 (1995).

[31] K.A. Olive, Proceedings of the 33rd International Winter School on Nuclear and Particle Physics, "Matter Under Extreme Conditions", Feb. 27 - March 5 1994, Schladming, Austria, eds. H. Latal and W. Schweiger (Springer-Verlag, Berlin, 1994) pp.1-37.

[32] D.V. Nanopoulos and K.A. Tamvakis, Phys. Lett. $110 B$ (1982) 449;

D.V. Nanopoulos, K.A. Olive and K.A. Tamvakis, Phys. Lett. 115B (1982) 15;

A.D. Dolgov and A.D. Linde, Phys. Lett. $116 B$ (1982) 329.

[33] I. Affleck and M. Dine, Nucl. Phys. B249 (1985) 361.

[34] M. Fukugita and T. Yanagida, Phys. Lett. B174 (1986) 45.

[35] J. Ellis, D.V. Nanopoulos and K.A. Olive, Phys. Lett. B300 (1993) 121.

[36] G.D. Coughlan, W. Fischler, E.W. Kolb, S. Raby and G.G. Ross, Phys. Lett. B131 (1983) 59. 
[37] J. Ellis, C. Kounnas and D.V. Nanopoulos, Phys. Lett. $143 B$ (1984) 410.

[38] J. Ellis, D.V. Nanopoulos and M. Quiros, Phys. Lett. B174 (1986) 176.

[39] J. Ellis, D.V. Nanopoulos and K.A. Olive, Phys. Lett. B184 (1987) 37.

[40] T. Moroi, M. Yamaguchi and T. Yanagida, Phys. Lett. B342 (1995) 105.

[41] R.L. Leigh and R. Rattazzi, RU-94-100 hep-ph/9503402 preprint (March, 1995). 


\section{FIGURES}

FIG. 1. Thermal mass correction to the gravitino mass. Crosses denote insertions of the soft masses for the matter and gluino fields. The dashed line represents a matter scalar field, the solid lines matter fermion and gluino fields.

FIG. 2. Thermal Goldstino vertex correction. The blobs denote thermal corrections to the gluino-gluon-Goldstino and to the matter fermion-scalar-Goldstino couplings.

FIG. 3. Thermal correction to the Goldstino self-energy. The blobs in the propagators are thermal self-energies for the gluon and gluino respectively. The blob is at the gluino-gluon-Goldstino vertex, which is the same as the zero-temperature vertex, as argued in Section 2.

FIG. 4. Two contributions to the Goldstino self energy. (a) For hard thermal loops, the resummed gluon propagator is the dominant effect. (b) For soft thermal loops, thermal corrections to the gluino and to the vertex are the dominant effects. 